\title{
Yrast phenomena in fashion
}

\section{The invention of a word new in the past decade signals a resurgence of interest in nuclear physics. But this time the objectives, if attainable, could be important.}

LEXICOGRAPHERS have been dismayed, but word-game players no doubt delighted, by the appearence on the scene of the word yrast, one of the rare contributions of the Swedish language to international usage. (The word is apparently the superlative of the adjective $y r$, which means "dizzy".) Its currency within the past decade reflects the interest which now centres on a recently unfashionable field of physics - the study of heavy nuclei by means of particle probes whose energies are modest by the standards set in high-energy physics laboratories, a few hundred $\mathrm{MeV}$ or thereabouts. Only last month, the general assembly of the European Science Foundation was congratulating itself on what had been accomplished, at least in the construction of specialized machines, in Europe in the past decade.

That the time is ripe for a resurgence of nuclear physics should not be a surprise. During the 1940s, interest in the properties of heavy nuclei was stimulated by the applications of nuclear fission then in vogue. (Thirty years ago, neutron cross-sections with heavy nuclei were still national secrets.) But the experience of that decade was itself sufficient to confirm what many people had expected, that the formal treatment of any but the simplest nuclei as systems of several interacting particles would be even more difficult than the calculation of the electronic structure of all but the simplest atoms.

Three developments have brought the field to life - the recognition that, with quarks and all that, nucleons are even more complicated but much more interesting than had been thought, heavy nuclei turn out to have previously unexpected but intriguing properties and there have been instrumental developments of great potential, both in the production of new kinds of probes for nuclear structure and in the measurement of what happens.

The experience of the past few years has shown that the rotational properties of heavy nuclei are arresting, well worth the invention of a word such as yrast. That a nucleus, being an object, should be capable of rotating about some axis is natural enough, while its small dimensions ensure that the rotation will be quantized, with permissible values of the total angular momentum determined by some integral quantum number and by Planck's constant.

But what if the nucleus is constitutionally asymmetrical, not spherical but, say, mildly sausage-shaped (an oblate spheroid)? And what if the rotation of the nucleus as a whole is not around the remaining axis of symmetry but, rather, in a perpendicular direction? That nuclei in the ground state can be aspherical has been known for decades - isotopes of $\mathrm{Yb}$ or $\mathrm{Hf}$ are familiar examples. The underlying explanation lies in the Fermi statistics that control the collective behaviour of the nucleons.

The route to the recognition of the interest of such nuclei rotating about an axis which is not the surviving axis of symmetry involved the recognition that collisions between nuclei, perhaps to form a compound nucleus whose eventual product would be aspherical, could leave a nucleus in such a state that its rotational energy was comparable with the excitation energy separating one nucleonic state from another. A glance at one of the usual physics journals will show that people are regularly using rotating nuclei with angular momenta of 50,80 or more quanta.

Last month's report to the European Science Foundation by the working group on nuclear physics was introduced by its chairman, Professor T. Mayer-Kuckuk from the University of Bonn, by means of an enthusiastic slide-show of the interest of these nuclei. One of his arguments is that a rotating nucleus, small though it may be, is not in principle very different from more familiar objects made of nuclear matter and rotating rapidly, the neutron stars which make pulsating stars for example. Certainly it seems to be on the cards that the further study of rotating nuclei will yield some information about the collective motion of particles in the much larger objects in the sky.

On the nuclear scale, it is already clear that the rotational state of a nucleus and the state of its internal nucleonic excitation are not independent. In classical language, the orbits of nucleons are affected by centrifugal and Coriolis forces (responsible, among other things, for cyclonic and anticyclonic patterns of terrestrial weather) caused by the rotation. On one view, that which models the internal conditions of a nucleus analogous to a superconducting state in which the motion of particles is truly collective, there is even a chance that sufficiently energetic rotation could cause a phase transition to a different state.

This view of the standard heavy nucleus as a model of collective motion in a relatively simple system, a hundred or two of nucleons in which collective motion dominates, is new. It also accounts for the now fashionable search for collective vibrational states, some of which surprisingly consist simply of pure radial oscillations whose frequency points directly to the compressibility of nuclear matter. But collectivity is not all, and experience has shown that the rotation of some nuclei cannot be explained by a rotation of the object as a whole but rather requires that the angular momenta of individual nucleon orbits, considered separately, should be aligned.

In the years ahead, according to MayerKuckuk, the field will be further enlivened by the further development of novel nuclear probes. The availability of antiproton beams should make the nuclear equivalent of positronium possible. The use of strange kaons (strange particles in the quark sense) should make possible for the first time measurements of nucleon momenta near the centre of the nucleus. Relativistic ion beams (nowhere yet produced) may yield remarkably new kinds of energy transfer. One goal now firmly in people's minds is to use old-fashioned nuclear physics to test field theories of strong interactions hitherto thought accessible only by the high-energy physics people. Making a bag of quarks might even provide a microscopic simulation of the Big Bang, if ever there was such a thing.

The European Science Foundation's committee has been concerned not merely with what might be accomplished but with the arrangements that exist for keeping the field alive. Mayer-Kuckuk has made a survey of all facilities in Europe, with telephone and telex numbers as appropriate, together with a calculation of which countries in Europe support how many people in the field. West Germany has 20 nuclear physicists per million inhabitants, while Britain is a long way down the list with a mere eight.

On balance, however, it seems that the field will prosper and that the new word yrast will soon be on everybody's tongue. What can it mean? Half-way between a noun and an adjective to judge from its usage, the simplest occurrence (as in "yrast trap") refers to the case in which two states of a nucleus, at least one of which is excited, have different angular momenta but nearly equal energies, so that it becomes convenient to look for transitions from one to the other. Why not?

John Maddox 